\title{
LBS Mobile Learning System Based on Android Platform
}

\author{
Ya-Li ZHANG ${ }^{a}$ and Xiao-Kan WANG \\ ${ }^{1}$ Henan Mechanical and Electrical Vocational College, Zhengzhou, 451191
}

\begin{abstract}
In the era of mobile internet, PC-end internet services can no long satisfy people's demands, needs for App and services on mobile phones are more urgent than ever. With increasing social competition, the concept of lifelong learning becomes more and more popular and accepted, making full use of spare time to learn at any time and any place meets updating knowledge desires of modern people, Location Based System (LBS) mobile learning system based on Android platform was created under such background. In this Paper, characteristics of mobile location technology and intelligent terminal were introduced and analyzed, mobile learning system which will fulfill personalized needs of mobile learners was designed and developed on basis of location information, mobile learning can be greatly promoted and new research ideas can be expanded for mobile learning.
\end{abstract}

\section{Introduction}

With the constant development of mobile communication technology and the hardware support provided by smartphone for GPS, smartphone users' needs for location information are getting stronger every day, LBS is applied in various phones to increase rich experiences for user, the development and innovation of LBS are largely promoted, solid technical support is generated for LBS mobile learning based on Android platform. LBS usually consists of two parts: obtaining users' current location information through GPS positioning, base station positioning, WIFI positioning and etc.; provide related services to satisfy particular demands on basis of users' location information. The goal of mobile learning system is to build a circumstance in which learners can acquire knowledge at any time and any place, select proper learning contents according to their own needs and organize real-time communication with each other. There are connections and differences between mobile learning and online learning, the major difference is their applied technologies, mobile learning uses wireless communication technology which enables learners to learn without time and location constraints which online learners suffer, it is more flexible and freer. See Table 1 for comparison of characteristics of mobile learning and online learning.

\footnotetext{
${ }^{a}$ Corresponding author: zhang20021026@163.com
} 
Table 1. Comparison of Characteristics of Mobile Learning and Online Learning

\begin{tabular}{|l|l|l|}
\hline Characteristics & Mobile Learning & Online Learning \\
\hline Learning Place & Any time and any place & Fixed Time and Place \\
\hline Network Environment & Wireless Network/4G & Wired Network \\
\hline Learning Mode & Online/Offline Learning & Online Learning \\
\hline Learning Device & Smartphone, iPad and etc. & Computer \\
\hline Communication & Any time and any place & Fixed Time and Place \\
\hline Interaction Easiness & $\begin{array}{l}\text { Relatively Limited } \\
\text { People-device Interaction }\end{array}$ & $\begin{array}{l}\text { Easy People-device } \\
\text { Interaction }\end{array}$ \\
\hline
\end{tabular}

Combining characteristics of mobile learning, a mobile learning platform which changes with a learner's location was developed on basis of LBS, real-time location circumstance was obtained on Android platform of system client-end, real-time check-in, content push, help, sharing and other functions of mobile learning system were performed, Java programming technology was used, encapsulation and implementation of XML data document interface were realized in Eclipse environment, data were built and services were offered, and eventually, LBS mobile learning system based on Android platform was realized.

\section{Selections on platform development}

Compared with other systems, Android system has properties like open source, rich hardware equipment, no limitation and etc.; Android architecture is divided into four levels from top to bottom: Application Level, Application Framework Level, Support Base Level and Linux Kernel Level; Android application development mainly comprises four components: Activity, Service, Content Provider and Broadcast Receiver. Each component has its own purpose. Activity Component provides visible interaction interface for user and program; Service Component implements time-consuming operations on background of main thread; Content Provider Component provides a group of unified interfaces for storing and reading data to realize data sharing among different programs; Broadcast Receiver Component receives broadcast messages which meet certain conditions. Moreover, Android SDK also provides nice-looking basic map services. Developers can refer to specific API documentation and develop particular LBS applications which satisfy their own needs. Only by completing relative configurations and integrating SDK into own applications, developers can realize positioning function through LBS positioning interface provided by Android SDK. Therefore, application developments based on Android is the prior choices for realization of client-end of this system. See Figure 1 for positioning principle of Android SDK.

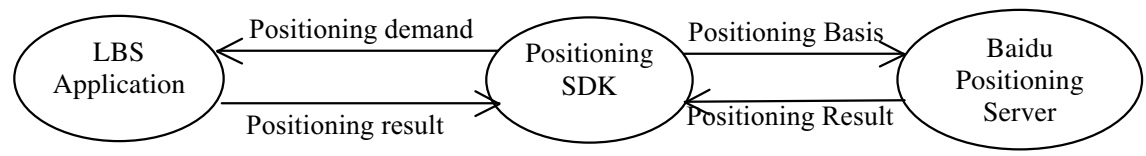

Figure 1. Positioning Principle of Android SDK

\section{LBS mobile learning system based on android platform}

\subsection{Analysis of system mode}

With the fast development of internet and information technology, organizing and obtaining modes of learning sources change continuously, mobile learning enters a new stage of circumstance perception from former single E-learning mode, exploring more on technology, resource, learning and 
circumstance of internet era is beneficial for innovation of mobile learning mode. Mobile learning based on LBS is centered with real current location and circumstance information of learners, guided by actual learning activities of learners, real-time location and circumstance information are integrated, learning contents and information helps which satisfy information demands and circumstance interaction are provided for learners. See Figure 2 for information interaction model built based on location perception.

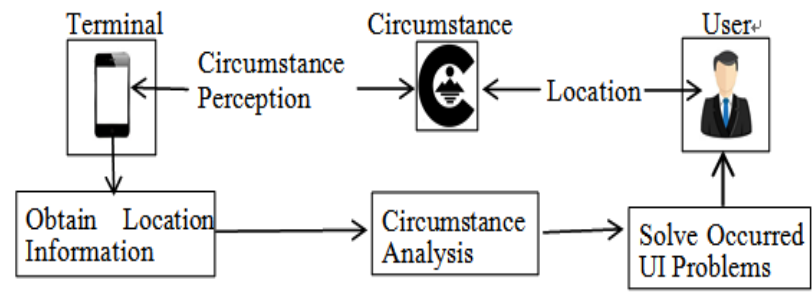

Figure 2. Circumstance Perception Mode Based on Location

\subsection{System data push technology}

Pushing data for learners is an important section in LBS mobile learning system based on Android platform. The push technologies commonly applied are: push based on association rules, push based on content, collaborative filtering push and etc., push based on content is used in the System. Items which users may perceive and push are presented by keywords, and weights of such keywords can be calculated with TF-IDF Method. Assume n-dimensional vector $\vec{d}_{k}=\left(W_{1 k}, W_{2 k}, \cdots, W_{i k}, \cdots, W_{n k}\right)$ is item, $\vec{d}_{l}=\left(W_{1 l}, W_{2 l}, \cdots, W_{i l}, \cdots, W_{n l}\right)$ is tendency of User $\mathrm{I}, \mathrm{n}$ is the number of keywords. According to TF-IDF Method, $T F_{i j}$ value definition of keyword $K_{i}$ in item set $d_{i}$ is as shown in Formula (1):

$$
T F_{i j}=\frac{f_{i j}}{\max _{z f_{z j}}}
$$

$I D F_{i}$ value definition of keyword $K_{i}$ in item set $d_{l}$ is as shown in Formula (2):

$$
I D F_{i}=\log \frac{N}{n_{i}}
$$

Final Weight Formula is obtained combining Formula (1) and (2), as shown in Formula (3):

$$
W_{i j}=T F_{i j} \cdot I D F_{i}=\frac{f_{i j}}{\max _{z f_{z j}}} \cdot \operatorname{lob} \frac{N}{n_{i}}
$$

"N" refers total number of item sets, $f_{i j}$ refers occurring times of keyword $K_{i}$ in item set $d_{k}$, $\max _{z_{f=1}}$ refers the maximum occurring times of keyword $K_{z}$ in all item sets, ${ }^{n_{i}}$ refers number of items in keyword $K_{i}$. 


\subsection{System design realization}

\subsubsection{Analysis of overall functional demands of the system}

The System consists of two parts: resource supplying service end and resource user end. On resource supplying service end, corresponding support service platform was designed. Positioning accurately and obtaining terminal location circumstance and other function modules are required for client-end of the System, in order to assure successful learning, functions listed in Table 2 shall be available on client-end:

Table 2. Basic Functions on Client-end of LBS mobile learning system based on Android platform

\begin{tabular}{|c|l|}
\hline Function Module & \multicolumn{1}{c|}{ Function Realization } \\
\hline User Registration & $\begin{array}{l}\text { Register basic information of users, follow and record their learning } \\
\text { situations. }\end{array}$ \\
\hline User Sign In & $\begin{array}{l}\text { Users voluntarily report their location circumstances, the System } \\
\text { automatically perceive users' location circumstances. }\end{array}$ \\
\hline Circumstance Learning & Users use local resources to learn. \\
\hline Learning Partner Sharing & $\begin{array}{l}\text { Position users' locations, search for surrounding learning partners } \\
\text { to share learning experiences, establish learning community. }\end{array}$ \\
\hline
\end{tabular}

\subsubsection{System service architecture}

The System applies a mixed system architecture of $\mathrm{C} / \mathrm{S}$ and $\mathrm{B} / \mathrm{S}$, its service end is API resource support service platform based on LBS, its client-end consists of devices which can connect with network, including mobile device and PC. As shown in figure 3, learning resources, location, platform service and other function modules are integrated on service end (cloud end) through data interface (open API), resource services are provided for mobile devices, PC and other client-ends through internet.

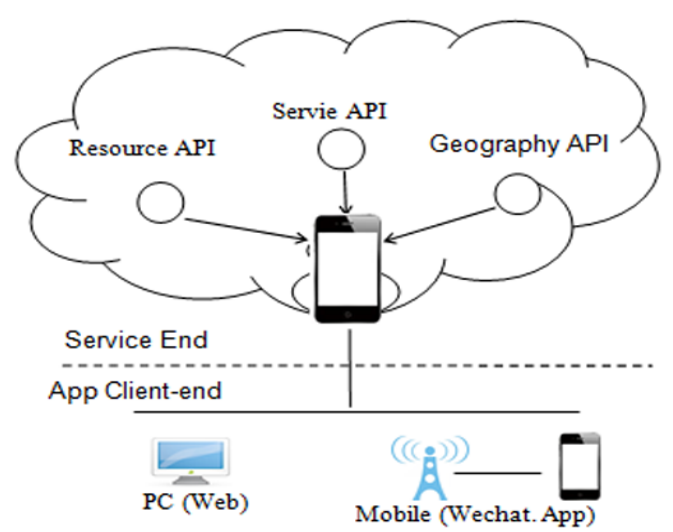

Figure 3. Architecture of Mobile Learning Service Based on Android Platform

\subsubsection{Key technologies for realization of client-end functions of the system}

There are two key technologies for realization of client-end functions of the System: The first is Android development technology; Android allows developers combine Internet data and local data, break distinctions between applications, third-party software can be conveniently called during 
client-end development process, assistant and support learning functions can be realized. Android provides application developers with a large number of local databases and utilities with which developers can establish their applications quickly. Android system is widely applied by mobile phone producers because of one of its characteristics -- open resource. The second is client-end network communication technology; there are mainly three communication modes between client-end and the server: HTTP, Socket and Web Service. In HTTP communication technology, client-end sends HTTP request to the server, the server receives the request from client-end, manages it and then responds client-end through HTTP; in Socket communication technology, external communication port is established inside application, while writing server-end and client-end codes, open the port and make communication protocol, verify safety and legality of data, after establishing connections, Socket directly realizes data communication without sending request to server-end; Web services are a kind of services based on XML and HTTPS technologies, they are deployed on Web server and managed by Web server. In order to offer satisfying user experiences, the most suitable communication mode will be selected according to demands of the System's transmitting data in realization of the System.

\subsubsection{Function modules of client-end of the system}

(1) User Registration Module

When learners start the application on mobile end, it will be checked if they ever registered or bind with basic information about their personal learning situations, if they never registered or bind, registration interface or binding interface will appear for them to fill in their basic learning situations. On one hand, bases can be provided to push personalized learning resources for learners; on the other hand, learning progresses of learners can be recorded continuously to provide bases for learning effects and evaluations.

(2) User Sign-in Module

User Sign-in Module is a process in which learners voluntarily report their location circumstances, acquire learning resources according to their location circumstances and learn online. In this module, it will be checked first if learners sign in as registered users, if not, it will jump to registration page. If users already registered and sign in successfully, mobile devices will automatically position locations of learners and report to the Platform, intelligent retrieval will be realized through perception reasoning, mobile client-end interface will be updated according to specific situations of mobile devices, knowledge will be presented in front of learners.

(3) Local Circumstance Resource Learning Module

One of the characteristics of Local Circumstance Resource Learning Module is downloading resources and storing them in local during idle hours of network. To-be-downloaded resources depend on pre-cached learning situations or pre-subscribed classified resources of learners. In environments with no or poor network, learners can retrieve resources fast for learning on client-end.

(4) Learning Partner Sharing Module

The client-end supports learning partner sharing function and learning community establishment. After obtaining and reporting current locations of learners, the information will be presented in form of POI data on real-time map, such POI information includes the problems which learners come across in learning, as well as their learning understandings and experiences, learner users within a certain range centered by learners will be searched, learning partnerships and community relationships can be established, real-time discuss and communication can be realized.

\section{System test}

On basis of System Design Realization Plan, in integrated development environment Eclipse, basic framework of service platform was realized through configuration files, basic functions were realized through writing organization codes, necessary function tests and application were conducted for prototype system. From aspect of operation and availability of the System, the Test was divided into two stages: the first stage is test of network resources and existing resources; the second stage is joint 
debugging of client-end and port. According to requirements of API closed design, ports are closed for resources which can be searched on platform, so that such resources can be called from client-end, resource sharing can be realized, resources can be retrieved by resource ID, resource open ports which are closed as SML data can be obtained through GET. "Key design codes of architecture ports which are closed as XML data" are as shown below.

public ComResource getResourceByIds(String resourceId)

í

ComResource com = resDao.getResourceById(resourceId);

if $(\mathrm{com}==$ null $)$

\{

return null;

\}

return com;

?

When users start client-end of mobile learning application based on LBS for the first time, current running environment of the Software will be checked, including network connection status, system positioning situation and whether it is the first time of using by users, initial data resources will be loaded.

\section{Conclusion}

In this Paper, LBS and currently popular mobile learning modes were combined, LBS mobile learning system based on Android platform was proposed. Mobile communication technology, LBS technology and mobile learning studies were analyzed, plan of mobile learning system based on LBS was established. It was suggested to apply LBS technology in mobile learning area and to integrate LBS and mobile learning. Application values of LBS in mobile learning were showed and improved, application of new technologies in education area was promoted to a certain extent.

\section{Acknowledgment}

This work was supported by the youth backbone teachers training program of Henan colleges and universities under Grant No. 2016ggjs-287, and the project of science and technology of Henan province under Grant No. 172102210124.

\section{References}

1. Yin Xixiang, Zhu Xianyuan, Chen Weiwei, "Design and Realization of Teaching Evaluation System Based on Android Platform" [J]. "Journal of Xinxiang University: Natural Science Edition”, 2014, 31(2):31-34.

2. Qiu Bingfa,“ Research and Design of Mobile Learning System Based on LBS”[D].Chongqing Normal University, 2016.

3. Xiong Shuirou, "Design and Realization of Learning System Based on Android Platform" [D]. Beijing University of Posts and Telecommunications, 2012.

4. Yang Jia, "Location Based Service of Mobile Learning System Design"[J]."Chinese Educational Informational",2012,07:31-33

5. Kinshuk,Rory McGreal,Sun Hongtao,Shi Pengfeng,Compilation."Mobile Learning System Based on Position to Identify 5R Adaptive Framework"[J]."Modern Distance Education Research",2012,03:91-96 
6. Zhang Gaoming,Ma Li,Zhao Jing."Campus LBS Application Research Based on Android Platform"[J]. "Journal of Xi 'an Liberal ArtsCollege: Natural Science Edition".2013,03 